Rev. salud pública. 13 (3): 528-540, 2011

Revisión/Review

\title{
Resiliencia en la vejez
}

\section{Resilience in old age}

\author{
Andrea Cárdenas-Jiménez y Alba L. López-Díaz \\ Facultad de Enfermería, Universidad Nacional de Colombia. Bogotá. acardenasj@unal.edu.co, \\ allopezdi@bt.unal.edu.co
}

Recibido 22 Agosto 2010/Enviado para Modificación 20 Mayo 2011/Aceptado 14 Junio 2011

\section{RESUMEN}

El objetivo del presente estudio fue caracterizar la literatura sobre resiliencia y vejez publicada en el período comprendido entre 1990 a 2006. Tras la búsqueda sistemática en cinco bases de datos (Academic Search Premier (Ebsco Host), Medline, PsycArticles, Ovid y ScienceDirect) se incluyeron en el análisis 33 piezas de literatura en las cuales se encontró un total de 31 definiciones diferentes para la resiliencia, surgidas de ocho disciplinas, principalmente de campos relacionados con la salud. Igualmente se encontró que los trabajos de investigación estudiaron la asociación de la resiliencia con factores individuales (68 variables) y con factores sociales/ambientales (17 variables). Los factores más frecuentemente estudiados fueron la edad y la autopercepción de salud. De especial interés entre los factores socio/ambientales son los valores culturales y religiosos. La revisión de literatura realizada, pone en evidencia que la resiliencia en la vejez es un tópico de creciente interés investigativo al que han sido asociados diferentes factores individuales, sociales y culturales, sin embargo se trata de un área en pleno desarrollo que requiere del establecimiento de una definición unificada, y de la creación de un modelo teórico y de intervención.

Palabras Clave: Resiliencia psicológica, envejecimiento, revisión (fuente: DeCS, BIREME).

\section{ABSTRACT}

The aim of this study was to characterise and analyse articles published on resilience and old-age from 1990-2006. After a systematic search of five databases (Academic Search Premier-Ebsco Host, Medline, Psyc Articles, Ovid and Science Direct) 33 pieces of literature were included in the analysis. The selected articles had 31 different definitions of resilience, from eight disciplines, mainly healthrelated fields. It was also found that the research studied the association of resilience with individual (68 variables) and social/environmental factors (17 variables); the most frequent were age and health self-perception. Cultural and religious values were of special interest amongst the latter variables. The literature review demonstrated that resilience in old age is a topic having increasing research interest; this has been linked to various individual, social and cultural factors. 
However, this is a rapidly developing area that requires that a unified definition be established and that a theoretical and intervention model be created.

Key Words: Resilience, psychological, aging, review (source: MeSH, NLM).

$\mathrm{L}$ a distribución por edades de la población mundial está pasando por una profunda transformación. A medida que las tasas de mortalidad y fertilidad han ido descendiendo, la distribución por edades se ha ido modificando gradualmente, impulsando la "transición demográfica" $(1,2)$. Aumentándose así, no solamente la expectativa de vida sino la representación de población vieja al interior de la población general. De esta manera, en la actualidad (y a futuro) no solo hay más personas viejas sino que ellas son ahora mucho más viejas.

La vejez es un período de retos adaptativos dado el cambio gradual de las condiciones de salud física y mental, las dificultades para el desarrollo de las actividades de la vida diaria y las restricciones a la participación social (3-6). Condiciones a las que se suman, las limitaciones en el acceso a los servicios sociales y de salud (7). La conjunción de estos factores llevan a que una alta proporción de la población vieja se encuentre en situación de discapacidad y pobreza $(2,8,9)$. De acuerdo con la literatura, el grado de la discapacidad tiende a aumentar en la medida que la persona envejece (10-12).

Pese al complejo y sombrío panorama antes presentado, existen individuos que son capaces de desarrollar procesos que les permiten afrontar, adaptarse y prosperar de cara a situaciones tan estresantes y adversas como pueden ser el envejecimiento en sus aspectos individuales, sociales y simbólicoculturales. A estos procesos sociales e intrapsiquicos se les conoce como resiliencia $(6,50)$.

Los estudios cualitativos que documentan la presencia de resiliencia en personas ancianas en situaciones de pobreza en Sao Paulo (Brasil) y en Bogotá (Colombia) (14-16), se constituyen en los antecedentes directos del presente estudio, desarrollado por el Grupo de Investigación Cuidado Cultural de la Salud de la Facultad de Enfermería de la Universidad Nacional de Colombia. Este estudio se desarrolló en el marco de un macroproyecto entre cuyos objetivos se encontraba indagar si existía resiliencia en un grupo de personas ancianas en situación de discapacidad y pobreza habitantes de la región central de Colombia (17-19). En la primera fase del proyecto se inició una revisión detallada de los antecedentes teóricos, conceptuales y empíricos 
de la resiliencia en la vejez (20). Es desde esta preocupación que el presente artículo busca caracterizar la literatura sobre resiliencia y vejez publicada en el período comprendido entre 1990 a 2006, en términos de su origen disciplinario y geográfico, frecuencia de publicación a lo largo del tiempo y metodología implementada para su desarrollo. Igualmente, se buscaba evidenciar las tendencias teóricas desde las cuales es conceptualizada la resiliencia y sus variables asociadas.

\section{MÉTODOS}

Para alcanzar los objetivos propuestos en el estudio se desarrolló una revisión sistemática de literatura, para la cual se exploraron cinco bases de datos: Academic Search Premier (Ebsco Host), Medline, PsycArticles, Ovid y ScienceDirect. Los términos utilizados en la búsqueda fueron "resilience and aging", "resilience and elderly" y "resilience and old". La búsqueda fue limitada a artículos de texto completo publicados entre 1990 y 2006, que contuvieran la palabra resiliencia o sus conceptos relacionados (hardiness, thriving, envejecimiento exitoso) en el título, las palabras clave, y/o el resumen.

De los 161 artículos emergentes de la búsqueda inicial 86 no fueron considerados por pertenecer a campos del saber como la física, la biología, las neurociencias o la ingeniería. Mediante la lectura de los resúmenes de las restantes 75 piezas se evalúo el cumplimiento de los restantes criterios de inclusión: hacer referencia en su objetivo general al estudio, teórico o empírico, de la resiliencia en la vejez o de sus conceptos relacionados; presentar una definición de resiliencia en la vejez y/o ser una investigación empírica, cuantitativa o cualitativa, o una revisión teórica. Una vez ejecutado este proceso se encontró que 33 artículos respondieron a todos los criterios de inclusión.

Las 33 piezas fueron analizadas mediante el uso de una matriz con seis secciones: procedencia del artículo (geográfica e institucional), definiciones de resiliencia o sus conceptos relacionados, objetivo del artículo, metodología, resultados y conclusiones.

\section{RESULTADOS}

Características de la literatura revisada

El desarrollo teórico y conceptual de la resiliencia en la vejez y la comprobación empírica reseñadas en los artículos que hicieron parte de la revisión han 
surgido en el marco de diferentes disciplinas tales como: la sociología, la gerontología, la geriatría, el trabajo social, la psiquiatría geriátrica, la medicina (social e interna), la enfermería y la psicología. Al interior de esta última se ha trabajado la resiliencia dentro de áreas tales como la psiconeuroinmunología, la psicología de la personalidad, la psicología genética, la psicología del desarrollo y el ciclo vital, la psicología social, la psicología del envejecimiento, la psicología humanista, la psicología positiva y desde el enfoque psicodinámico.

En términos geográficos, la producción es mayoritariamente de origen norteamericano (Estados Unidos y Canadá) lo cual se evidencia en el $72 \%$ de los artículos revisados (24 artículos), aunque vale resaltar la presencia de un $15 \%$ (5 artículos) de investigaciones europeas (alemanas, suecas e irlandesas), de $6 \%$ (2) del Medio Oriente (israelí), $3 \%$ de Oceanía (Australia) y $3 \%$ hecho con población taiwanesa. Llama la atención que ninguno de los artículos incluidos en la revisión proceda de América Latina o el Caribe. Esta falta de representación de la Región en la muestra de investigaciones sobre resiliencia en la vejez puede estar relacionada con el hecho de que no se hayan incluidos bases de datos latinas en las búsquedas iníciales. Sin embargo, también debe considerarse que la producción latinoamericana y caribeña se encuentra escasamente representada en las publicaciones y bases de datos internacionales.

En relación con el número de artículos según año de publicación se encontró que existe una tendencia creciente con el paso del tiempo. Pues mientras que al inicio del periodo considerado (1990-2006) se publicaron muy pocos artículos, el primero en 1993, dicho número creció encontrándose un notorio aumento en las publicaciones a partir del año 2000, momento a partir del cual se publicó el $90 \%$ de los artículos incluidos en la revisión. En los dos últimos años del periodo (2005 y 2006) se publicaron 13 artículos, es decir el $40 \%$ del total revisado. Este hallazgo podría estar relacionado con el creciente interés que la resiliencia ha suscitado como campo de investigación e intervención entre profesionales de diversas disciplinas y con el descubrimiento de la importancia que su rol desempeña durante la tercera edad.

El $27 \%$ (9 artículos) de la literatura revisada correspondió a artículos que abordaban cuestiones teóricas, conceptuales y metodológicas relacionadas con la resiliencia en la vejez, mientras que el $73 \%$ (24 artículos) presentaba trabajos de investigación. 
Dentro de las piezas investigativas la metodología utilizada fue en gran porcentaje $(79 \%$ ) de tipo cuantitativo encontrándose estudios observacionales principalmente (18 de los 19 estudios) entre los cuales el $56 \%$ fueron transversales y el $44 \%$ longitudinales (5 estudios prospectivos y 3 retrospectivos). En menor proporción se encontró el abordaje cualitativo el cual fue implementado en un $21 \%$ (5 estudios) de los trabajos de investigación revisados, en los cuales básicamente se utilizaron entrevistas en profundidad como técnica de recolección de datos y diferentes técnicas de análisis de datos, entre las cuales cabe mencionar la Grounded Theory, el método fenomenológico y la triangulación de datos, esta última en el estudio de Fry (29) que combinó las metodologías cualitativa y cuantitativa.

Definiciones de Resiliencia

Dentro de las 33 piezas de literatura revisada se encontraron 31 definiciones explícitas y diferentes del concepto de resiliencia. Dichas definiciones, de forma global se caracterizaron por su heterogeneidad en cuanto a enfoques teóricos, manifestaciones y resultados. Sin embargo, todas las definiciones de resiliencia encontradas tienen una estructura común, caracterizada por la presencia de cuatro elementos: el tipo de atributo como la plantea cada definición, sus manifestaciones, las dimensiones de la vida en las que se evidencia y las demandas ambientales ante las cuales se pone de manifiesto.

Según el tipo de atributo se encontró que la resiliencia es concebida, mayoritariamente, como habilidad o capacidad personal (12 definiciones), como característica positiva de la personalidad. (3 definiciones), como proceso transaccional (4 definiciones) y/o como capacidad adaptativa (4 definiciones). Además, se encontraron tendencias menos frecuentes, tales como aquellas que plantean que la resiliencia puede ser concebida como conocimientos, insights, capacidad fisiológica, respuesta, o de manera más general, como un fenómeno humano. Es de resaltar la presencia de una definición en que la resiliencia se plantea como la ausencia de factores negativos más que como la presencia de factores positivos.

Las manifestaciones de resiliencia presentes en las definiciones que nos ocupan fueron clasificadas, para la presente revisión, en cuatro grupos. En primer lugar, aquellas que hacen referencia a la conservación de los recursos con que cuentan los individuos antes de los eventos adversos; entre ellas se encuentran "mantenerse bien", "sobrevivir" y "resistir". En un segundo grupo están aquellas manifestaciones en las que se evidencia que los individuos recuperan los recursos alterados, modificados o perdidos como efecto del 
evento adverso; entre ellas pueden incluirse la "recuperación", la "restauración y el "resurgimiento". En tercer lugar se encuentran los resultados positivos que los individuos pueden construir a partir de la adversidad; de este modo según las definiciones los individuos pueden "prosperar", "crecer", "mejorar", "superarse", "ir más allá", "fortalecerse" y "transformar el desastre en experiencias de crecimiento". En el último grupo de manifestaciones resilientes se encontrarían los procesos mediante los cuales sería posible el logro de los resultados previamente mencionados, vale destacar entre ellos el "afrontamiento", el "ajuste" y la "adaptación".

El tercer componente de las definiciones, es decir, las dimensiones de la vida en las que se evidencia la resiliencia incluye principalmente la salud física y/o emocional, el estatus funcional, las capacidades, el balance, el envejecimiento exitoso y la calidad de vida. Por último, el cuarto componente de las definiciones, el referente a las demandas ambientales ante las cuales se pone de manifiesto la resiliencia en la vejez, abarca diversas experiencias negativas entre las que cabe mencionar la adversidad y las experiencias adversas, las alteraciones de la salud en general, y las enfermedades devastadoras y las limitaciones físicas mayores, en particular. Las experiencias negativas también incluyen las pérdidas vitales mayores, el estrés, los daños potenciales, la amenaza acumulada, los retos, las experiencias difíciles y el riesgo.

Conceptos relacionados con la resiliencia

A lo largo de la revisión se encontró que junto a la resiliencia en la vejez diferentes autores plantean una serie de conceptos cercanamente relacionados pero no plenamente diferenciados de ella. En general, dichos conceptos pueden ser vistos como parte de dos amplias concepciones:, la primera, como productos propios del desarrollo que permiten una mejor vivencia del proceso de envejecimiento y la segunda, como interacciones entre las características individuales y las demandas ambientales.

El concepto más representativo dentro de la primera concepción es el "envejecimiento exitoso" para el cual es posible afirmar que, al igual que con la resiliencia, no existe consenso acerca de su definición, sin embargo, se reconoce entre sus componentes la longevidad, la satisfacción, el bienestar, la resiliencia, la ausencia de enfermedad física, la ausencia de discapacidad, el dominio, el compromiso activo con la vida, la vida independiente y la adaptación positiva (48). 
En la segunda concepción se encuentran el "hardiness" y el "trhiving", conceptos cuya traducción más cercana, pero no por ello más precisa, sería "fuerza" y "crecimiento". El "hardiness" es definido como una "característica de la personalidad que modera la relación estrés-enfermedad y que está conformado por el deseo de mantenerse activo, la creencia de que uno influye sobre el curso de los eventos vitales, y la creencia de que el cambio puede ser un estimulo para el crecimiento" (24-27); y el "thriving", se plantea como respuesta al estrés que lleva a resultados mentales, físicos y sociales positivos. El thriving, también llamado crecimiento post-traumático, está relacionado exclusivamente con los resultados positivos del estrés, mientras que la resiliencia considera la severidad de los eventos negativos y el grado de recuperación tanto como los nuevos efectos positivos del evento (6).

Es de resaltar que aunque en la revisión realizada se encontró la definición de otros diez conceptos relacionados con la resiliencia (adaptación, dominio del estrés, envejecimiento transformativo, bienestar subjetivo, bienestar psicológico, estrategias de ajuste cognitivo, sentido de coherencia, autotrascendencia, propósito en la vida, optimismo y esperanza), los tres que fueron definidos son no solo los más cercanos a la resiliencia, conceptualmente hablando, sino que, en el caso del hardiness y del envejecimiento exitoso, son los más frecuentemente estudiados en las investigaciones empíricas revisadas.

Factores asociados con la resiliencia

De los estudios empíricos analizados, tanto cuantitativos como cualitativos, surgen diversos hallazgos según los cuales la resiliencia en los viejos se encuentra asociada con un conjunto de factores que potencian y posibilitan los resultados resilientes de cara a los eventos adversos propios de la vejez. Dichos factores, que pueden catalogarse como individuales y sociales / ambientales, son de naturaleza diversa y hacen parte de múltiples dimensiones del ser humano, los cuales son abordados de forma parcial por cada estudio.

En relación con los factores individuales, se encontró que su asociación con la resiliencia ha sido estudiada de forma| mucho más frecuente que la de los factores sociales / ambientales, de forma que dentro de la literatura revisada se investigó un total de 68 variables de tipo individual diferentes, mientras que tan solo se trabajo con 17 variables de tipo social / ambiental. Los factores individuales pueden ser categorizados como demográficos, emocionales, cognitivos, de personalidad, relacionados con el afrontamiento, relacionados con la salud y el funcionamiento, y relacionados con la autopercepción. Dentro de dichas variables las más frecuentemente estudiadas fueron la auto- 
percepción de salud (7 artículos), el estado de salud física (6 artículos), la presencia de síntomas depresivos ( 5 artículos) y el estrés (3 artículos).

En cuanto a los factores sociales / ambientales los estudios reportan la relación de la resiliencia con factores familiares, de soporte social extra familiar, culturales, históricos, relacionados con el afrontamiento y relacionados con el acceso a servicios de cuidado de la salud. Dentro de este grupo de variables las más estudiadas fueron el apoyo social y la religiosidad con 4 artículos, respectivamente.

Al revisar los hallazgos de los estudios cualitativos se destaca que los valores culturales influyen sobre la resiliencia en los viejos por medio de su efecto sobre las formas de ver el mundo propias de cada cultura $(7,25,28,29)$. De especial importancia en el marco de lo cultural es la religiosidad como factor fuertemente asociado con la resiliencia en los viejos, específicamente en lo relativo a las creencias y la asistencia a los servicios religiosos $(4,7,25,30)$.

En términos generales, se encontró que uno de los tópicos más contradictorios dentro de los hallazgos revisados es el relacionado con el nivel de resiliencia en la vejez según el género. Pues mientras que algunos autores reportan que existen diferencias, entre hombres y mujeres, a favor de unos $(4,5,28,30$,$) , u otras (33), hay quienes no encuentran diferencia alguna$ (26). De forma más consensuada los estudios revisados señalan que los factores que subyacen a la resiliencia se conservan hasta el final del ciclo vital $(37,42)$ y que incluso van en aumento a medida que los viejos se hacen más viejos, lo cual se evidencia al realizar comparaciones entre grupos de diferentes edades (5). Sin embargo, el estudio de Rothermund y Brandtstadter (36) demostró que la resiliencia a los síntomas depresivos disminuye a medida que avanza la edad.

Finalmente, estudios como el de Wallace. (27) encontraron asociaciones significativas entre la resiliencia y algunos de sus conceptos relacionados, (previamente enumerados). De esta manera se ha encontrado asociación entre la resiliencia y la autotrascendencia, el propósito en la vida, el sentido de coherencia $(5,34)$, y el envejecimiento exitoso (35). Igualmente, Maddi \& Hightower (43) encontraron asociación con el optimismo, mientras que Ong (40) lo hizo con la esperanza. 


\section{DISCUSIÓN}

En la actualidad no existe consenso respecto a la definición de resiliencia en la vejez, lo cual lleva a que cada estudio se desarrolle de acuerdo con una definición y una metodología (diseños e instrumentos) particulares, dificultándose de esta manera la comparabilidad entre los hallazgos de cada uno. Esta tendencia podría estar relacionada con el creciente interés que con el paso del tiempo el tema de las fortalezas y factores positivos individuales ha despertado entre autores de diferentes disciplinas y áreas de conocimiento y con la riqueza y gran potencialidad que tiene la resiliencia como campo de investigación. El hecho de que en las definiciones se aborden elementos concretos como las manifestaciones de resiliencia y las demandas ambientales ante las cuales se hacen evidentes favorece la búsqueda de un concepto unificado y el desarrollo de programas de fortalecimiento de la resiliencia basados en él, que sean evaluables y replicables con poblaciones diversas. La relevancia de dichos programas radica en que su fomento se ha constituido en uno de los objetivos principales de las Políticas de Promoción en Salud Mental (23).

Como ha podido apreciarse, la investigación en resiliencia tiene una fuerte inclinación hacia el nivel individual de análisis. La mayoría de las investigaciones y los artículos conceptuales definen la resiliencia, como expresión del temperamento, como fenómeno interno o psíquico. Este hecho es fiel reflejo de su origen en el campo de la psicología (49). Así, pocos investigadores han explorado las características de la resiliencia que surgen de sistemas como la familia, la comunidad o los valores culturales (46). Sin embargo, existe un reconocimiento creciente de que la resiliencia es multidimensional y multideterminada (13,51). Esta perspectiva es relativamente nueva en el estudio de la resiliencia, e invita al esfuerzo interdisciplinario para explorar la articulación entre los procesos psicológicos y aquellos del ambiente no psicológico $(47,49)$.

La predominancia de investigaciones cuantitativas con interés preponderante en los atributos intraindividuales, puede estar relacionada con la mayor representación de investigaciones de origen (geográfico e intelectual) norteamericano, cuya corriente teórica es de tipo conductista, pragmática e individualista (50), mientras que las investigaciones de tipo social / comunitario, de origen latinoamericano, no hicieron parte de esta revisión. 
Según los hallazgos de los estudios cualitativos revisados en este trabajo, es posible señalar que las técnicas utilizadas en ellos son de especial importancia para la investigación en resiliencia dado que se ajustan a la complejidad del fenómeno, al considerar el contexto social, histórico y cultural en el cual se desarrolla y al tomar en consideración las variables subjetivas $(20,21,50)$. Por esta razón es importante el desarrollo de nuevos estudios cualitativos, dada su baja representatividad en el universo de la investigación en resiliencia.

Muchos de los estudios revisados consideraban como variables de resultado la salud y el bienestar, encontrándose que se recuperaban, se mantenían y mejoraban en los ancianos resilientes. Hallazgos como estos abren la posibilidad de demostrar a los proveedores de servicios de salud la importancia que tiene el fortalecimiento de los aspectos psicológicos y específicamente de aquellos relacionados con la resiliencia para la construcción, mantenimiento y restauración de la salud y calidad de vida en la población adulta mayor. Sin embargo, es necesario tomar en consideración las implicaciones que traen consigo las estrategias de promoción de la resiliencia dado que ellas no sustituyen las acciones encaminadas a la mejora de las circunstancias de vida objetivas de las personas.

Como aporte para la investigación futura, el presente estudio plantea la necesidad de nuevas revisiones con criterios de búsqueda más restringidos que permitan establecer parámetros de comparación entre los diferentes estudios. Igualmente se requieren estudios de metanálisis que permitan evaluar la fuerza de las asociaciones reportadas por el cuerpo de investigación sobre resiliencia en la vejez, lo cual podría contribuir a la construcción de una definición unificada del concepto.

El principal aporte de la revisión de literatura realizada, para la cual, dicho sea de paso no se encontraron antecedentes, es haber puesto en evidencia que la resiliencia en la vejez es un tópico de creciente interés investigativo al que han sido asociados diferentes factores individuales, sociales y culturales. Sin embargo se trata de un área en pleno desarrollo que requiere del establecimiento de una definición unificada, de la creación de un modelo teórico y de intervención dirigido a la identificación y fortalecimiento de factores positivos más que a la reducción de los negativos y del desarrollo de estudios cualitativos que permitan conocer los factores subjetivos subyacentes y la influencia percibida de los factores contextuales * 
Agradecimientos: Con el apoyo financiero del Instituto Colombiano para el Desarrollo de la Ciencia y la Tecnología. COLCIENCIAS y la Facultad de Enfermería de la Universidad Nacional de Colombia, código: 11010416453; La Dirección de Investigación Sede Bogotá, Universidad Nacional de Colombia- DIB, código: 20601003556.

\section{REFERENCIAS}

1. Department of Economic and Social Affairs of the United Nations. World Economic and Social Survey 2007. Development in an Ageing World. New York; 2007.

2. Gasparini L, Alejo J, Haimovich F, Olivieri S, Tornarolli L. Poverty among the elderly in Latin America and the Caribbean. Argentina: CEDLAS; 2007.

3. Debert G. A reinvenção da velhice. Socialização e processos de reprivatização do envelhecimento. São Paulo: Edusp; 2004.

4. Consedine NS, Magai C, Krivoshekova Y. Sex and age cohort differences in patterns of socioemotional functioning in older adults and their links to physical resilience. Ageing International. 2005; 30(3):209-244.

5. Nygren B, Jonsen A, Gustafson Y, Norberg A, Lundman B. Resilience, sense of coherence, purpose in life and self-transcendence in relation to perceived physical and mental health among the oldest old. Aging \& Mental Health. 2005; 9(4):354-362.

6. Hardy S, Concato J, Gill T. Resilience of Community-Dwelling Older Persons. JAGS. 2004; $52,57-262$.

7. Becker G, Newsom E. Resilience in the Face of Serious Illness Among Chronically III African Americans in Later Life. The Journals of Gerontology. 2005; 60(4):S214-23.

8. DANE. [Internet] Boletín Censo General 2005. Discapacidad Colombia. Actualización. Disponible en: http://www.dane.gov.co/censo/files/boletines/discapacidad.pdf Consultado en: Julio 17 de 2010.

9. Huenchuan S, Guzmán JM. Seguridad económica y pobreza en la vejez: tensiones, expresiones y desafíos para el diseño de política, Revista Notas de Población. 2007; 83, (LC/G.2340-P):99-125.

10. Ruipérez I. Envejecimiento, siglo XXI y solidaridad. Rev Esp Geriatr Gerontol. 2002; 37 (Suppl 2):3-7.

11. Organización Mundial de la Salud-Grupo Orgánico de Enfermedades No Transmisibles y Salud Mental Departamento de Prevención de las Enfermedades No Transmisibles y Promoción de la Salud. Envejecimiento y Ciclo Vital. Envejecimiento activo: un marco político. Rev Esp Geriatr Gerontol. 2002; 37(Suppl 2): 74-105.

12. WHO. Innovate care for chronic conditions. Geneva: World Health Organization; 2002.

13. Walsh F. Fortalecendo a Resiliencia Familiar. Sao Paulo: Roca; 2005.

14. López AL, Cianciarullo TI. Compreendendo o significado de qualidade de vida na velhice. Texto Contexto Enferm. 1999; 8(3):233-49.

15. López AL. Revelando o oculto cuidar de pessoas idosas: uma proposta para a promoção da igualdade de gênero. En: Blay, Org, Igualdade de Oportunidades para as Mulheres En: São Paulo: Humantias FFLCH/USP; 2002.

16. Lopez AL. Cianciarullo, TI. A velhice e a qualidade de vida: um olhar cultural. En: Gualda DM, Bergamasco RB, Org. Enfermagem, cultura e o processo saúde-doença. São Paulo: Ícone Editora; 2004.

17. López AL, Alzate ML, Velásquez V. Prácticas de cuidado realizadas por personas ancianas en situación de discapacidad y pobreza en zona rural y urbana de Colombia. In: X Coloquio Panamericano de Investigación en Enfermería. Memorias y Programas; 27-30; Buenos de Aires, Argentina. Encuentro Grupo Editor; v.1; 2006. p. 146. 
18. López L, Alzate M, Velásquez V. Poverty, disability and old age in Colombia: a cultural care perspective. In: 33rd Annual conference of the Transcultural Nursing Society. Bournemouth University. Abstract Book; 2007 Sep 15-21; Bournemouth, Inglaterra; p. 24.

19. Castellanos F, López L. Significados de la discapacidad de los ancianos en un contexto de pobreza. III Congreso Iberoamericano de Investigación Cualitativa en Salud. Escuela Graduada de Salud Pública, Recinto de Ciencias Médicas, Universidad de Puerto Rico. Libro de Resúmenes; 2008 May 6-9; San Juan, Puerto Rico; pp. 57-58.

20. Cárdenas A, López L. Resiliencia ante la vejez, la discapacidad y la pobreza: historia oral de vida. III Congreso Iberoamericano de Investigación Cualitativa en Salud. Escuela Graduada de Salud Pública, Recinto de Ciencias Médicas, Universidad de Puerto Rico. Libro de Resúmenes; 2008 May 6-9; San Juan, Puerto Rico; p. 122-123.

21. Ong A, Bergeman C. Resilience and adaptation to Stress in later life: Empirical perspectives and Conceptual implications. Ageing International. 2004; 29(3): 219-246.

22. Schoon I, Binner J. Risk and Resilience in the Life Course: Implications for Interventions and Social Policies. Journal of Youth Studies. 2003; 6 (1):21-31

23. Organización Mundial de la Salud. Promoción de la Salud Mental: Conceptos, evidencia emergente, práctica. Informe compendiado. Ginebra: OMS; 2004.

24. Langer N. Resiliency and spirituality: foundations of strengths perspective counseling with the elderly. Educational Gerontology. 2006; 30:611-617.

25. Felten B. Resilience in a Multicultural Sample of Community-Dwelling Women Older Than Age 85. Clinical Nursing Research. 2000; 9(2):102-123.

26. Keneally P. Hardiness, self-care practices and perceived health status in older adults. Journal of advanced nursing. 1993; 18:10 5-1094.

27. Wallace KA, Bisconti T, Bergeman CS. The Mediational Effect of Hardiness on Social Support and Optimal Outcomes in Later Life. Basic \& Applied Social Psychology. 2001; 23(4):267-279.

28. Hardy S, Concato J, Gill T. Stressful Life Events Among Community-living Older Persons. Journal of General Internal Medicine 2002; 17(11):841-847.

29. Fry P. Spousal loss in late life: a 1-year follow-up of perceived changes in life meaning and psychosocial functioning following bereavement. Journal of Personal \& Interpersonal Loss. 1998; 3(4):369-391.

30. Walter-Ginzburga A, Shmotkina D, Blumsteinb T, Shoreka A. A gender-based dynamic multidimensional longitudinal analysis of resilience and mortality in the old-old in Israel: the cross-sectional and longitudinal aging study (CALAS) Social Science \& Medicine. 2005; 60:1705-1715.

31. Montross L, Depp C, Daly J, Reichstadt J, Golshan S. Moore D, Sitzer D, Jeste D. Correlates of Self-Rated Successful Aging Among Community-Dwelling Older Adults. Am J Geriatr Psychiatry. 2006; 14(1)

32. Seplaki C, Goldmana N, Weinsteinb M, Linc Y. Before and after the 1999 Chi-Chi earthquake: Traumatic events and depressive symptoms in an older population. Social Science \& Medicine. 2006; 62:3121-3132.

33. Walker C. Transformative Aging: How Mature Adults Respond to Growing Older. Journal of Theory Construction \& Testing. 2002; 6(2): 109.

34, Schneider G, Driesch G, Kruse A, Nehen H, Heuft G. Old and III and Still Feeling Well? Determinants of Subjective Well-Being in $>60$ Year Olds: The Role of the Sense of Coherence. Am J Geriatr Psychiatry. 2006; 14(10).

35. Wagnild G. Resilience and Successful Aging: Comparison Among Low and High Income Older Adults. Journal of Gerontological Nursing 2006; 29(12): 42-49.

36. Rothermund K, Brandtstädter J. Depression in Later Life: Cross-Sequential Patterns and Possible Determinants. Psychology and Aging 2003; 18(1): 80-90.

37. Jopp D, Root C. Adaptation in Very Old Age: Exploring the Role of Resources, Beliefs, and Attitudes for Centenarians' Happiness. Psychology and Aging 2006; 21(2):266-280. 
38. Kahana E, Lawrence R. Kahana B, Kercher K, Wisniewski A, Stoller E, Tobin J, Stange K. Long-Term Impact of Preventive Proactivity on Quality of Life of the Old-Old. Psychosomatic Medicine. 2002; 64:382-394.

39. Roberts C, Cleveland L. Surrounded by Ocean, A World Apart. The Experience of Elder women Living Alone. Holist Nurs Pract. 2001; 15(3):45-55.

40. Ong A, Edwards L, Bergeman C. Hope as a source of resilience in later adulthood. Personality and Individual Differences. 2006; 41:1263-1273.

41. Ong A, Bergeman C, Bisconti T, Wallace K. Psychological Resilience, Positive Emotions, and Successful Adaptation to Stress in Later Life Journal of Personality and Social Psychology. 2006; 91(4):730-749.

42. Hamarat E, Thompson D, Steele D, Matheny K; Simons C. Age Differences in Coping Resources and Satisfaction With Life Among Middle-Aged, Young-Old, and OldestOld Adults. Journal of Genetic Psychology 2002; 163 (3):360.

43. Maddi S, Hightower M. Hardiness and Optimism as Expressed in Coping Patterns. Consulting Psychology Journal: Practice and Research 1999; 51(2):95-105.

44. Bonanno G, Wortman C, Nesse R. Prospective Patterns of Resilience and Maladjustment During Widowhood. Psychology and Aging 2004; 19 (2):260-271.

45. Pinquart M. Good News About the Effects of Bad Old-Age Stereotypes. Experimental Aging Research 2002; 28(3):317-336.

46. Ehrensaft E, Tousignant M. Ecología Social y Humana de la Resiliencia. En: Manciaux M. La resiliencia: Resistir y rehacerse. Barcelona: Gedisa; 2003.

47. Waller M. Resiliece in Ecosystemic Context: Evolution of the Concept. American Journal of Orthopsychiatry $2001 ; 7(3): 290-297$.

48. Depp C, Jeste D. Definitions and Predictors of Successful Aging: A Comprehensive Review of Larger Quantitative Studies. Am J Geriatr Psychiatry. 2006; 14(1).

49. Manciaux M. La resiliencia ¿mito o realidad? En: Manciaux M. La resiliencia: Resistir y rehacerse. Barcelona: Gedisa; 2003.

50. Suárez E. Resiliencia y Subjetividad. En MelilloA, Suarez E, Rodriguez D. (Comp.). Resiliencia y Subjetividad. Los Ciclos de la Vida: Buenos Aires: Paidos; 2004.

51. Cárdenas A, López AL. Analysis Matrix of Resilience in the Face of Disability, Old Age and Poverty', International Journal of Disability, Development and Education. 2010; 57 (2):175-189. 